\title{
TENSILE DAMAGE MECHANISM OF 3-D ANGLE-INTERLOCK WOVEN COMPOSITE USING ACOUSTIC EMISSION EVENTS MONITORING
}

\author{
Qian Ma ${ }^{1 \dagger}$, Ke Wang ${ }^{1 \dagger}$, Shu-Dong Wang ${ }^{1}$, Hua Liu' ${ }^{1}$, Bo-Cheng Jin², Li-Min Jin ${ }^{3,4,5 *}$, Pibo Ma ${ }^{6,7 *}$ \\ ${ }^{1}$ Jiangsu Research and Development Center of the Ecological Textile Engineering and Technology, College of Textile and Clothing, Yancheng Vocational \\ Institute of Industry Technology, Yancheng 224005,P.R. China. \\ ${ }^{2}$ Gill Composites Center, Department of Chemical Engineering and Materials Science, University of Southern California, Los Angeles, CA 90089, USA. \\ ${ }^{3}$ Shanghai Institute of Applied Physics, Chinese Academy of Sciences, Shanghai 201203,P.R.China. E-mail: Imjin@mail.dhu.edu.cn \\ ${ }^{4}$ College of Textiles, Donghua University, Shanghai 201620, P.R.China. \\ ${ }^{5}$ Hubei Key Laboratory of Advanced Textile Materials \& Application, Wuhan Textile University, Wuhan 430200, China. \\ ${ }^{6}$ Key Laboratory of Eco-textiles, Ministry of Education, Jiangnan University, Wuxi 214122, P.R. China. E-mail: mapibo@jiangnan.edu.cn \\ ${ }^{7}$ Engineering Research Center for Knitting Technology, Ministry of Education, Jiangnan University, Wuxi 214122, P.R. China \\ * Corresponding author \\ ${ }^{\dagger}$ These authors contributed equally to this work.
}

\begin{abstract}
:
The quasi-static tensile damage behavior of one type of layer-to-layer 3-Dimensional Angle-interlock Woven Composite (3DAWC) was tested and analyzed in this paper. Incorporated with the acoustic emission (AE) events monitoring, the mechanical behavior of the 3DAWC under tensile loading condition was characterized. The LoadExtension curve, Load/AE events-Time curves occurred during the entire testing process and tensile damage modes were recorded to characterize and summarize the mechanical properties and damage mechanism of the 3DAWC subjected to tensile loading. It was found that the tensile damage of the 3DAWC could be summarized into 3 steps. And each step has a distinct primary damage mode. Moreover, the resin cracks, resin-yarn interface debonding and yarn breakages were the main damage modes for the 3DAWC.
\end{abstract}

\section{Keywords:}

3-D Angle-interlock Woven Composite (3DAWC), Quasi-static tension,Acoustic Emission (AE),Damage mechanism

\section{Introduction}

Three-dimensional (3D) textile structural composites (3DTSCs) are currently being widely used in structural engineering fields. [1] As one typical type of 3DTSCs, the 3D Angle-interlock Woven Composites (3DAWCs) have attracted increasing interest during recent years due to their excellent mechanical properties. Angle interlock weaving is a technique in which yarns are placed at an angle to the thickness direction.[2, 3] Such weaving structures induce high damage tolerance and energy dissipation performance along the thickness direction for the 3DAWCs. Taking these significant advantages of the 3DAWCs into account, the research works performed on the mechanical properties and structural optimization of the 3DAWCs have become of critical importance.

To design the 3DAWCs meet the requirements of engineering applications, firstly their mechanical behavior and damage mechanism need to be analyzed. So far, the mechanical behaviors of the 3DAWCs have been investigated and analyzed by several researchers and their co-workers. Sheng et al.[4] proposed a 3D micromechanical modeling approach to predict the elastic constants of 3DAWC, good agreement was obtained between the predicted results and experimental results. Sun et al. $[5,6]$ investigated the compressive properties of the layer-tolayer 3DAWC at quasi-static and high strain rate loading using the methods of Fourier transform and wavelet packet analysis of stress waves to evaluate the compressive failure modes at different strain rates. It revealed that the stress-strain curves of the 3DAWC were sensitive to strain rate. Lapeyronnie et al.[7] assessed the elastic behavior of the layer-to-layer 3DAWC by an asymptotic homogenization procedure on a periodic unit cell, in the framework of the Love-Kirchhoff plate theory. They developed a specific Python program using ABAQUS software package, allowing for parameterized geometrical modeling and mechanical analysis in a systematic and efficient way. The effective properties were finally validated by comparison with experimental tests. Dong et al.[8] established a finite element model of the layer-to-layer 3DAWC, which truly simulate the profile and tending towards of tows in fabric. The model was adopted to study the mechanical properties of the composites. And the influence of the arranged density of the warp and weft tows on the elastic moduli of this material were also analyzed. It showed that the models were found to correlate reasonably well with the experimental, predicted and measured results available in the literature. Tan et al.[9] used a laminate block modeling approach for through-the-thickness 3DAWC to develop one finite element analysis (FEA) model and two analytical models. These models were used to determine the mechanical properties and the coefficients of thermal expansion for the composites. A parametric study showed that there was a good agreement between these FEA and analytical models. Nehme et al.[10] proposed a new numerical FEA and analytical model to evaluate the mechanical behavior of the layer-to-layer 3DAWC. A good agreement between these two models was also obtained. Also, Hallal et al.[11] developed an 
analytical model to estimate the longitudinal Young's modulus of the layer-to-layer 3DAWC.

Considering the excellent de-lamination resistance performance because of the existence of undulated warp yarns along the thickness direction, the 3DAWCs have also been applied in high-velocity impact-proof protection. Several researchers have performed research works on ballistic penetration behavior of the 3DAWCs to explore the failure mechanisms under impact damage. Cui et al.[12] and Li et al.[13] employed a 3D finite element "Fiber inclination model"and unit-cell model to simulate the ballistic penetration process and summarize the energy absorption and damage mechanisms of a layerto-layer 3DAWC, respectively. Tang et al.[14] characterized the microstructure, impact response, and failure modes of the 3DAWC with cellular structures under transverse impact with both experimental investigations and finite element unit-cell modeling. A good agreement was found between FEA results and experimental results.

As one of the main issues for composite materials in structural engineering applications, fatigue behavior analyses are widely employed because of the requirement of safe applications. Considering the excellent performance and wide range of potential applications for the 3DAWCs, understanding their fatigue behavior is of critical importance. As for the fatigue behavior of the 3DAWCs, Tsai et al.[15] conducted a comparative study on the fatigue properties and damage processes between the untouched three-layer and five-layer 3DAWC plates under tensile cyclic loading. The fatigue damage mechanisms primarily involved transverse cracks in the warp yarns, debonding between the warp and weft yarns, debonding extension and deflection into the matrix and weft yarn breakages. Gowayed et al.[16] developed a model that was established from a mathematical model to analyze the fatigue behavior and life of fiber/epoxy 3DAWC; the obtained results were in good agreement with the experimental data. More recently, Jin et al.[17-19] studied the three-point bending fatigue behaviors of the layer-tolayer 3DAWC. It was found that the integrated construction structure can resist the de-lamination of the 3DAWC under high-cycle fatigue loading. In addition, 3DAWC shows a better bending fatigue resistance performance compared with the 3D Orthogonal Woven Composites (3DOWC) at the same stress levels.

However, the damage mechanism of the 3DAWCs under tensile loading has not been properly analyzed;to design stronger and more effective 3DAWC structures, more active effects should be made. In this paper, the damage mechanism of one type of the layer-to-layer 3DAWCs subjected to quasi-static tension loading is reported. The tests were performed with Acoustic Emission (AE) monitoring technique. The Load-Extension curve, Load/AE eventsTime curves were obtained to characterize the mechanical behavior of the 3DAWC under quasi-static tensile loading. Moreover, the AE events occurred during the entire testing process and ultimate failure modes were illustrated to unveil the damage mechanism of the 3DAWC under quasi-static tension loading.

\section{Experimental}

\subsection{Materials}

Figure 1 is the sketch diagram of alayer-to-layer 3D Angleinterlock Woven Fabric (3DAWF) construction. In this structure, the undulated warp yarns interlaced the non-crimp weft yarns to form a stable and integrated woven construction. All the warp yarns and weft yarns were glass fiber tows. Table 1 lists the specifications of the 3DAWF.

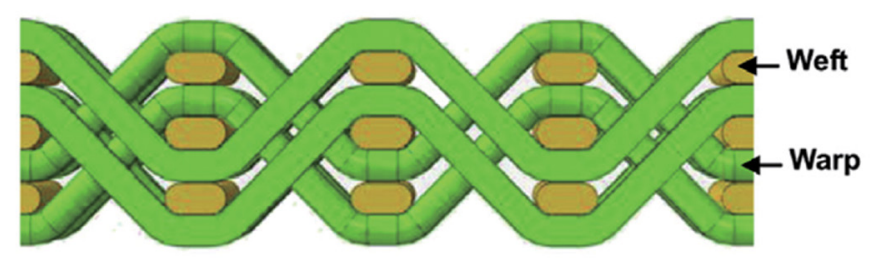

Figure 1. Layer-to-layer angle-interlock structure

Table 1. Specifications of the3DAWF

\begin{tabular}{|c|c|c|c|c|}
\hline Yarns & $\begin{array}{c}\text { Fiber } \\
\text { type }\end{array}$ & $\begin{array}{c}\text { Linear } \\
\text { density } \\
\text { (Tex) }\end{array}$ & $\begin{array}{c}\text { Density } \\
\text { (ends/ } \\
\text { cm) }\end{array}$ & layers \\
\hline Warp & $\begin{array}{c}\text { glass } \\
\text { fiber tows }\end{array}$ & $480 \times 2$ & 11 & 5 \\
\hline Weft & $\begin{array}{c}\text { glass } \\
\text { fiber tows }\end{array}$ & $480 \times 1$ & 2 & 6 \\
\hline
\end{tabular}

The vacuum assisted resin transfer molding (VARTM) technique was employed to manufacture the 3DAWC. The resin was AROPOLTM INF 80501-50 polyester resin provided by Ashland Composite Polymers China. The curing agent was $A K Z O \circledR$ $M-50$. The proportion of resin and curing agent was 100:1.0 by weight. The resin solution was first injected into the 3DAWF and then cured for 24 hours at a room temperature of $25^{\circ} \mathrm{C}$ followed by a post curing in an oven at $80^{\circ} \mathrm{C}$ for 4 hours. The 3DAWC samples used for testing were cut with high pressure water jet along the longitudinal and transverse directions of the composite plate. The longitudinal direction of the samples was aligned with the warp yarns. In all the quasi-static tension tests, to avoiding the occurrence of stress concentration effects at the clamping regions, which may induce the irregular local damage, the dog-bone shaped testing specimens were manufactured and used. Thus, it ensures that tensile fracture failure occurs at the central part of the specimen. The size (length $\times$ width $\times$ thickness) of each 3DAWC sample was $12 \times 1.87 \times 0.4 \mathrm{~cm}$. The fiber volume fraction of the 3DAWC was approximately $55 \%$. The surface and cross-section of a sample are shown in Figure 2.

\subsection{Quasi-static tension tests}

As shown in Figure 3, the quasi-static tension tests were conducted on an INSTRON 8501 system at the speed of $1 \mathrm{~mm} /$ min. Moreover, in order to monitor the damage development of the composite sample during the entire testing process, an acoustic emission (AE) system (Physical Acoustics PCl-2) was employed. Two $300 \mathrm{kHz}$ resonance transducers (Micro 
a

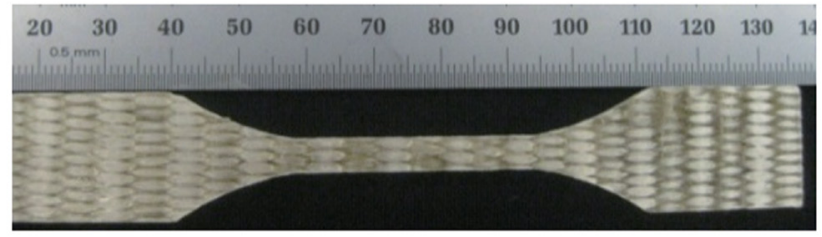

b

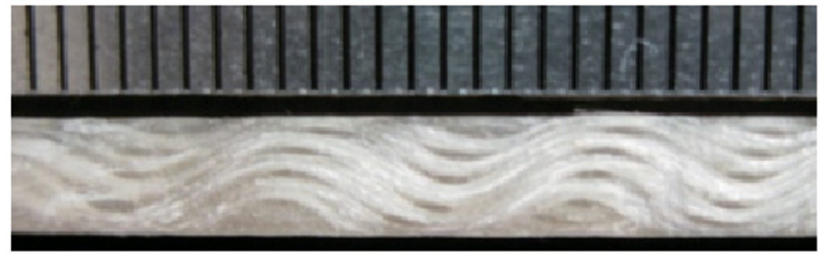

Figure 2. 3DAWC testing sample; (a) Surface, (b) Cross-section
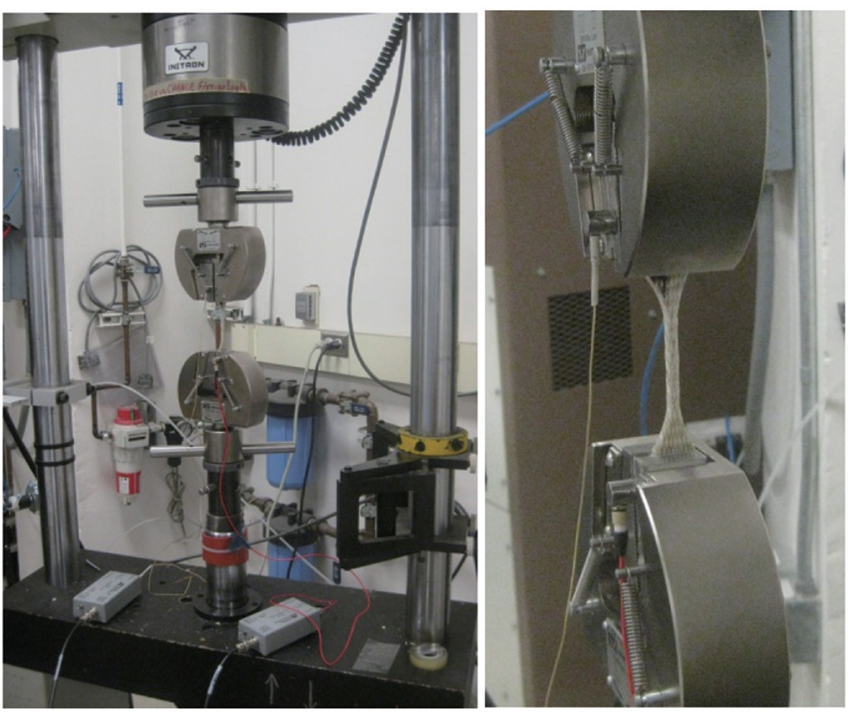

Figure 3.Testing setup

30) were placed on the loading fixtures to determine when and where the damage events took place. The sensors were attached to model 2/4/6 preamps providing $40 \mathrm{~dB}$ of gain and band-pass filtering of $200-400 \mathrm{kHz}$. The location of the $A E$ source in the composite specimen was determined using a linear location method. Only the AE events recorded between the sensors were used to analyze the results.

\section{Results and discussion}

\subsection{Load-extension and Load/AE events-time curves}

In order to determine the dominant failure mechanisms during the process of tensile loading, the load-extension curve, and load/number of AE events of the 3DAWC samples during the quasi-static tensile test are plotted together as a function of time and presented in Figures4 and 5, respectively.

It can be significantly found that both curves can be divided into 3 regions, that is, Regions I, II and III. For Region I, there is a sharp rise of both load and number of $A E$ events during a short period of testing time between 0 and 25th second. For Region II, between the 25th and 55th second, a continuous tiny

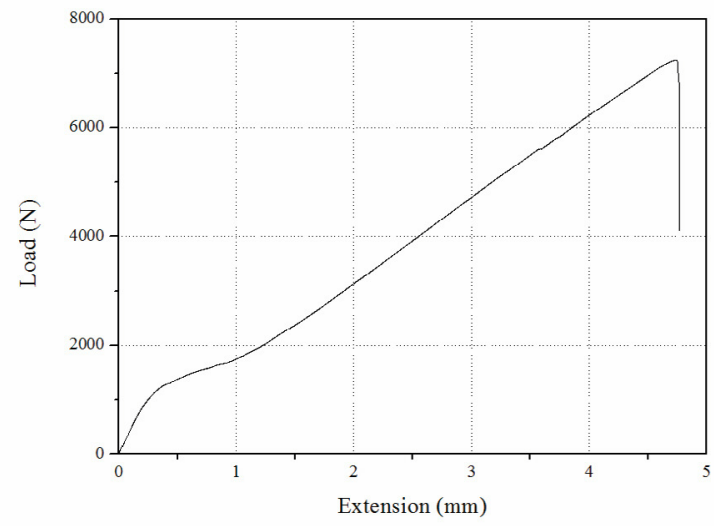

Figure 4. Load-extension curve

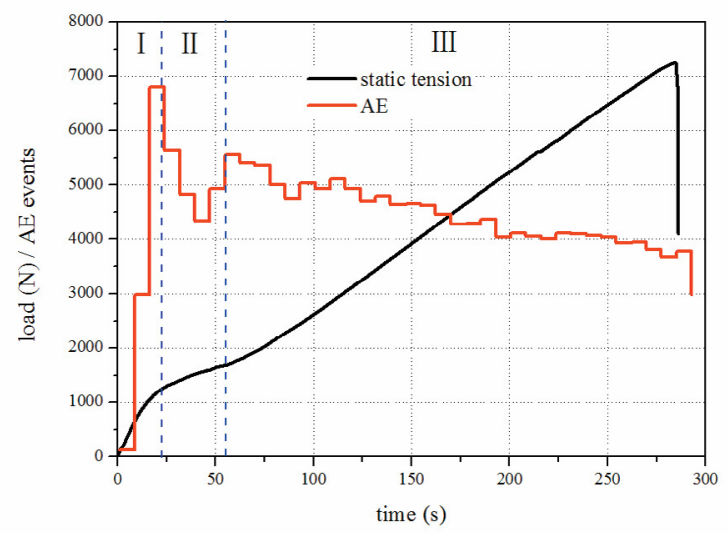

Figure 5. Load/AE events-time curves

rise for the load, and firstly a decrease and then an increase for the number of $A E$ events exist simultaneously. As for Region III, from 55th second to the ultimate failure, there is a continuous rise for the load and a continuous ladder-like increase and decrease, but generally shows a down-ward trend for the number of $A E$ events.

\subsection{Failure morphologies}

The ultimate failure morphologies of the 3DAWC sample under quasi-static tensile loading are presented in Figure 6, which shows the typical damage modes of the 3DAWC at both views of the surface. It can be found that the resin cracking, yarn breakages and pulling out from the matrix and breakages of undulated warp yarns are the main damage mode. In particular, the resin cracks and the yarn breakages can be easily found on the surface of the sample.This indicates that the undulated warp yarns carried majority of the loads during the static tensile loading. The debonding was relatively easier to occur at the stress concentration regions of warp yarn-resin-weft yarn interface, where the undulated warp yarns hold the maximum amplitude of undulation. Moreover, the damage is initiated from the edge part of the sample and then propagated into the inner structure. 


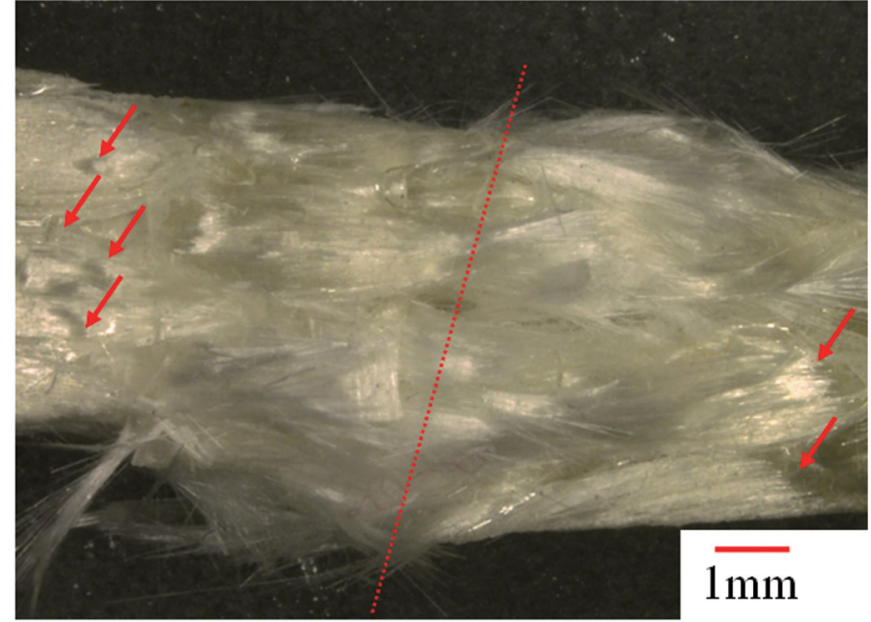

Figure 6. Damage morphology

\subsection{Damage mechanism}

Take the structural characteristic of the 3DAWC into consideration, the phenomena summarized above should be identified as the different dominant damage mechanism among these 3 regions.

For Region I, it was mainly attributed to the resin cracking After the tensile load was applied on the sample, at the area of finest central surface, the resin cracking began to occur after a short period of loading time. With the rise of load, the cracking propagated along the thickness direction of the sample. Such a process consumed a short period of the testing time and led to a sharp rise in the number of $A E$ events. Then, the process of damage entered Region II, the resin cracks continued to propagate into the inner layers of the composite. More importantly, the de-bonding at resin-yarns interface also took place, and simultaneously, the cracks propagated from the resin to the yarns as a result of the stress concentration. This region also took up a short period of loading time, during which a continuous tiny rise for the load occurred along with firstly a decrease and then an increase in the number of $A E$ events. As for Region III, it was primarily identified as the progressive breakages of yarns/fiber tows, especially the undulated warp yarns along the load-carrying direction of the 3DAWC sample. As the cumulative damage process of the specimen continued, the occurrence of resin damage and interfacedebonding reached a saturated state. And the gradual breakages of yarns became the primary damage mode in this stage.This process occupied a relatively long testing time, and therefore, a continuous ladder-like increase and decrease, but generally a downward trend for the number of $\mathrm{AE}$ events.

\section{Conclusions}

The quasi-static tension properties and damage mechanisms of the layer-to-layer 3DAWC were tested and analyzed. It is found that the load-extension and load-time curves of the composite samples show gradual and different amplitude increments in 3 stages, which means that each step has a distinct primary damage mode. Such phenomenon is in accordance with the results of the AE events' detection for characterizing the progressive damage of the composite. In addition, the undulated warp yarns, which ran throughthe thickness direction of the 3DAWC, plays an important role in carrying the loads.

\section{ACKNOWLEDGEMENTS}

This research was financially supported by the Open Project Program of Key Laboratory of Eco-textiles, Ministry of Education, Jiangnan University(No.KLET1505 ), a grant from the financial support by Jiangsu Overseas Research \& Training Program for University Prominent Young \& Middle-aged Teachers and Presidents (2014) and Natural Science Foundation of the Higher Education Institutions of Jiangsu Province(16KJB540005). The authors acknowledge the financial support from the China Postdoctoral Science Foundation (No. 2016M591767), the Applied Foundation Research Funds of China Textile Industry Association (J201604), and the Open Project Program of Hubei Key Laboratory of Advanced Textile Materials \& Application, Wuhan Textile University (Fzxcl2017001).

The authors are also grateful for the support extended for the project by Top-notch Academic Programs Project of Jiangsu Higher Education Institutions(PPZY2015C254) and Scientific Research Fund of Yancheng Institute of Industry Technology.

\section{References}

[1] Mouritz, A.P., Bannisterb, M.K., Falzonb, P.J., Leongb, K.H. (1999). Review of applications for advanced threedimensional fibre textile composites. Composites Part A, 30(12), 1445-1461.

[2] Chen, X., Spola, M., Paya, J.G., Sellabona, P.M.(1999). Experimental studies on the structure and mechanical properties of multi-layer and angle-interlock woven structures. Journal of the Textile Institute, 90(1),91-99.

[3] Chen, X., Potiyaraj, P. (1999).CAD/CAM of orthogonal and angle-interlock woven structures for industrial applications, Textile Research Journal, 69(9),648-655.

[4] Sheng, S.Z., Hoa, S.V. (2003). Modeling of 3D angle interlockwoven fabric composites. Journal of Thermoplastic Composite Materials, Journal of Thermoplastic Composite Materials, 16(1), 45-58.

[5] Sun, B.Z., Gu, B.H., Ding, X. (2005).Compressive behavior of3-D angle-interlock woven fabric composites at variousstrain rates. Polymer Testing, 24(4): 447-454.

[6] Sun, B.Z.,Gu, B.H. (2007).Frequency analysis of stress waves intesting 3-D angle-interlock woven composite at highstrain rates, Journal of Composite Materials, 41(24), 2915-2938.

[7] Lapeyronnie, P. Grognec, P.L., Binétruy, C., Boussu, F. (2011).Homogenization of the elastic behavior of a layerto-layer angle-interlock composite. Composite Structures, 93(11), 2795-2807.

[8] Dong, W.F., Xiao, J., Li, Y. (2011).Finite element analysis of the tensile properties of $2.5 D$ braided composites. Materials Science and Engineering A, 457(1-2),199204. 
[9] Tan, P., Tong, L.Y.,Steven, G.P. (1999).Micromechanics models for mechanical and thermomechanical propertiesof $3 D$ through-the-thickness angle interlock woven composites. Composites: Part A, 30(5),637-648.

[10] Nehme, S., Hallal, A., Fardoun, F., Younes, R.,Hagege, B.,Aboura, Z., Benzeggagh, M., Chehade, F.H. (2011). Numerical/analyticalmethods toevaluate the mechanical behaviorof interlock composites.Journal of Composite Materials, 45(16), 1699-1716.

[11] Hallal, A., Younes, R., Nehme, S., Fardoun, F. (2011).A corrective function for the estimationof thelongitudinal Young's modulus in a developed analytical model for 2.5Dwoven composites. Journal of Composite Materials, 45(17), 1793-1804.

[12] Cui, F., Sun, B.Z., Gu, B.H. (2010).Fiber inclination model for finite elementanalysis of three-dimensional angleinterlock woven composite underballistic penetration. Journal of Composite Materials, 45(14),1499-1509.

[13] Li, Z.J., Sun, B.Z., Gu, B.H., (2010).FEM simulation of 3D angle-interlock woven composite under ballistic impact from unit cell approach. Computational Materials Science, 49(1), 171-183.
[14] Tang, Y.Y., Sun, B.Z., Gu, B.H. (2010). Impact damage of $3 D$ cellular woven composite from unit-cell level analysis. International Journal of Damage Mechanics, 20(3),323346.

[15] Tsai, K.H.,Chiu, C.H.,Wu, T.H. (2000).Fatigue behavior of $3 D$ multi-layer angle interlock woven composite plates. Composites Science and Technology, 60(2),241-248.

[16] Gowayed, Y., Fan, H. (2001). Fatigue behavior of textile composite materials subjected to tension-tensionloads. Polymer Composites, 22(6), 762-769.

[17] Jin, L.M., Sun, B.Z., Gu, B.H. (2012).Three-point bending fatigue behavior of $3 D$ angle-interlock woven composite. Journal of Composite Materials, 46 (8), 883-894.

[18] Jin, L.M., Niu, Z.L., Jin, B.C., Sun, B.Z., Gu, B.H. (2012). Comparisons of static bending and fatigue damage between $3 D$ angle-interlock and $3 D$ orthogonal woven composites. Journal of Reinforced Plastics and Composites, 31 (14), 935-945.

[19] Jin, L.M., Yao, Y., Yu, Y.M., Rotich, G., Sun, B.Z., Gu, B.H. (2014).Structural effects of three-dimensional angleinterlock woven composite undergoing bending cyclic loading. Science China Physics, Mechanics \& Astronomy, 57(3), 501-511. 